Expert Opin Biol Ther. 2013 September ; 13(9): 1229-1240. doi:10.1517/14712598.2013.804507.

\title{
Cell-based therapy for ischemic stroke
}

\author{
Jing Zhang, MD PhD ${ }^{\dagger}, 1$ and Michael Chopp, PhD $^{1,2}$ \\ ${ }^{1}$ Department of Neurology, Henry Ford Health System, Education \& Research Building, \#3056, \\ 2799 West Grand Boulevard, Detroit, MI, 48202, USA \\ ${ }^{2}$ Oakland University, Department of Physics, Rochester, MI, 48309, USA
}

\begin{abstract}
Introduction-Stroke is a major cause of mortality and disability in adults worldwide.

Unfortunately, current therapy which targets vessel recanalization has a narrow treatment window, and at this time neuroprotective approaches are not effective for stroke treatment. However, after stroke the parenchymal and endothelial cells in the central nervous system (CNS) respond in concert to ischemic stressors and create a microenvironment in which successful recovery may ensue. Neurogenesis, synaptogenesis, axonal sprouting, glial cell activation, angiogenesis and vascular remodeling within the brain and the spinal cord are stimulated post stroke. Cell basedtherapy amplifies these endogenous restorative effects within the CNS to promote functional outcome.
\end{abstract}

Areas covered-This article reviews current knowledge of cell-based therapy in the adult brain after stroke, including transplanted cell type, benefits and risks, with an emphasis on mechanisms of action.

Expert opinion-Experimental studies and clinical trials with cell-based therapy in stroke appear promising. Cell-based therapy is not intended for the replacement of damaged cells, but for the remodeling of the CNS by promoting neuroplasticity, angiogenesis and immunomodulation. However, there are risks associated with the use of cell-based therapy, and adequate evaluation of these potential risks is a prerequisite before clinical application for stroke patients.

\section{Keywords}

cell-based therapy; neurorestoration; stem cell; stroke

\section{Introduction}

Stroke is one of the leading causes of death and disability worldwide. With an annual incidence of 250 - 400 in 100,000 inhabitants, many stroke survivors are left with permanent neurological disability. This leads to a huge social and economic burden. The majority of strokes are ischemic, caused by the interruption of blood flow in a brain-

\footnotetext{
(C) 2013 Informa UK, Ltd. All rights reserved

†Author for correspondence: Tel: +1 313916 3936; Fax: +1 313916 1318;jingz@ @euro.hfh.edu.

Declaration of interest

The authors declare that we have no conflict of interest.
} 
supplying artery from a thrombus or embolus. Stroke produces an ischemic core with irreversible damage, and is surrounded by a penumbra of surviving cells that highly influence the functional recovery. Pathophysiological responses in brain after stroke are complex and involve multiple mechanisms, including disturbance of neuronal circuitry, slowed cellular energy metabolism, and disruption of the blood-brain barrier (BBB) [1]. In addition, reperfusion post ischemia may worsen tissue damage by increasing inflammation and free radicals [2]. All these pathophysiological changes lead to neuronal and glial cell injury and death, axonal loss and demyelination with subsequent functional deficits and disabilities.

The currently available therapies of acute stroke target rapid vessel recanalization and neuroprotection, since without restoration of cerebral blood flow, tissue residing in the penumbral region progresses to cellular death which ultimately expands the core lesion. Recombinant tissue plasminogen activator (tPA) is the only drug approved by the Food and Drug Administration (FDA) for clinical stroke treatment. tPA is an enzyme that works by catalyzing the conversion of plasminogen to plasmin which can then lyse the embolus or thrombus causing the cerebral ischemia; therefore, tPA may restore cerebral blood flow in the penumbra and protect damaged ischemic cells from death. However, current utilization of tPA is very low, and fewer than $10 \%$ of stroke patients receive tPA in clinical practice, likely because of its narrow therapeutic time window (within $4.5 \mathrm{~h}$ of symptom onset) [3-5]. Moreover, a potential adverse effect of tPA treatment is hemorrhage [3]. Although there are potential targets for neuroprotective therapies that have shown promise under a narrow range of applications in animal stroke models, none has demonstrated clinical efficacy [6]. For example, preclinical findings suggest that erythropoietin (EPO) provides neuroprotection after ischemic stroke [7], $N$-methyl d-aspartate (NMDA) antagonists [8] and gamma-aminobutyric acid (GABA) potentiators [9] are used to block excitotoxic cell death; thrombin inhibitors reduce secondary thrombosis [10], and intercellular adhesion molecule 1 (ICAM-1) inhibitors reduce inflammatory reperfusion injury [11].

A more effective approach for the treatment of stroke would be enhance the endogenous restorative mechanisms of recovery, that is to treat, not the lesion, but to treat the intact CNS. Patients with stroke tend to improve. Yet we have failed to investigate the biological substrate underlying this improvement, and to capitalize on endogenous restorative processes post stroke. Exogenous cell-based treatments promote cerebral plasticity and neurological recovery after stroke. And cell-based therapies have emerged as promising options for treating stroke [12].

This review provides an account of cell-based therapy for stroke, and includes characterization of exogenously administered cells, and the evidence for therapeutic benefit and potential risks of the cell-based therapy derived from preclinical studies and clinical trials. In addition, we describe mechanisms underlying the therapeutic benefits of cell-based therapy on outcome improvement after stroke. 


\section{An array of cell-based therapy for stroke}

Cell-based therapy has been investigated as an alternative strategy to improve neurological outcome after ischemic stroke for more than a decade. Reports from preclinical rodent models of ischemic stroke and clinical trials using stem cells or adult and fetal progenitor cells have shown therapeutic promise. Stem cells are undifferentiated cells that have the capacity to proliferate (self-renew) and to differentiate into mature specialized cells. There are other candidates for cell-based therapy which do meet the precise definition of a stem cell as a self-renewing cell that can give rise to all cells in an organ, such as neural precursor cells (NPCs) that are obtained from the subventricular zone (SVZ) [13], endothelial progenitor cells (EPCs) that are isolated from the bone marrow [14] and human multipotent adult progenitor cells (hMAPCs) [15], among others.

Briefly, stem cell types include pluripotent stem cells that can give rise to all of the cell types that make up the body, and multipotent stem cells that can develop into more than one cell type, but are more limited than pluripotent cells. Pluripotent stem cells include embryonic stem cells (ESCs) and induced pluripotent stem cells (iPSCs) [16]. ESCs are derived from the inner cell mass of the blastocyst, which are totipotent cells to differentiate into derivatives of all three germ layers (endoderm, mesoderm and ectoderm) [17]. They have the ability to undergo unlimited proliferation in vitro [17]. iPSCs are artificially derived from adult differentiated somatic cells, such as fibroblasts, liver, pancreas $\beta$ cells and mature B cells, by "forced" expression of specific genes (i.e., Oct4, Sox2, c-Myc, Klf4, Nanog and Lin28) using somatic cell nuclear transfer (SCNT) method [18,19]. iPSCs have very similar growth characteristics, gene expression profiles, epigenetic modifications and developmental potential as ESCs [16]; therefore, this reprogramming breakthrough may make iPSCs clinically practical, and is a promising source of patient specific cells for use in regenerative medicine. Multipotent stem cells include fetal stem cells (FSCs), neural stem cells (NSCs) and mesenchymal stem cells (MSCs). FSCs are derived from birth tissues, that is amniotic fluid, placenta and umbilical cord blood [20]. The relatively easy accessibility and high proliferation rate make FSCs excellent cell sources for regenerative medicine. NSCs have been isolated from various areas of the adult brain and spinal cord; they generate mainly phenotypes of the nervous system (e.g., neurons, astrocytes and oligodendrocytes) [21]. MSCs are derived from various sources, such as bone marrow, placenta, cord blood, even adipose tissue [22,23]. Currently, the most extensive pre- and clinical experience has been obtained with MSCs.

Various cell types are candidates for cell therapy in ischemic stroke. The ideal cell for transplantation should meet all the criteria of safety, be relatively easy to obtain adequate numbers of cells for the treatment, as well as offer the highest therapeutic potential. Although ESCs offer an unlimited source of neural cells for repair after stroke [24], adult derived stem cells have become the cells of choice to study [25], primarily to avoid the ethical dilemmas of using ESCs and FSCs. Progress has been made in isolating human adult NSCs and demonstrating the feasibility of autologous transplantation [21]. These cells have shown positive therapeutic effects in several neurological disorders without causing serious side effects [25]. iPSCs reprogrammed from adult human somatic cells produce patientspecific cells for autologous transplantation. Oki found that transplantation of human iPSC- 
derived cells is a safe and efficient approach for the treatment of experimental stroke [26]. After transplantation of neuroepitheliallike stem cells that were generated from adult human fibroblasts, improved functional outcome was observed associated with increased vascular endothelial growth factor (VEGF) levels and enhanced endogenous plasticity. Moreover, these transplanted cells stopped proliferating and differentiated to mature neurons without forming tumors. MSCs provide a promising tool for cell therapy after ischemic stroke. The interest in MSC applicability for clinical treatment is based on their easy isolation and their great expansion capability, multipotential differentiation capacity [27] and extensive preclinical data indicating therapeutic efficacy. In addition, MSCs induce very weak or no immune reactions [28], and they are widely accepted by allogeneic transplantation or xenotransplantation in animal models. In 2001, the first studies of vascular transplantation of multipotent stem cells isolated from bone marrow [29] and umbilical cord blood cells (UCBCs) [30] were performed. These transplanted cells enter the brain, target the ischemic border, stimulate endogenous restorative effects and improve neurological functional recovery $[29,30]$.

\section{Mechanism underlying the benefits of stem cell therapy for stroke}

The mechanisms underlying the therapeutic benefits of transplanted cells on ischemic brain are being actively investigated. In response to ischemia, a series of factors are produced by brain parenchymal cells and endothelial cells and a microenvironment is initially stimulated by stroke to promote recovery [12].

Systemic cell infusion, that is intravenous injection, although noninvasive, leads to high uptake of cells in the liver, spleen, lungs and kidneys [31], with relatively few cells entering the ischemic brain. These transplanted cells migrate and communicate with endogenous cells via the stromal cell-derived factor-1 (SDF-1) and CXC chemokine receptor-4 (CXCR-4) axis [32], gap-junction coupling [33], among other factors.

Since few transplanted cells are found in the brain, and they have a very low capacity for neural transdifferentiation [34], exogenously administered cells do not directly replace the damaged tissue. The therapeutic benefit likely results from targeting and stimulating endogenous parenchymal cells, leading to enhancement of neural plasticity, including neurogenesis, angiogenesis, synaptogenesis, axonal sprouting and oligodendrogenesis [12,35-37], and modulation of inflammation [15] in the ischemic brain. Interactions among these restorative events contribute to the improvement in functional outcome (Figure 1), even though only a single infusion of stem cells was administered during the subacute or chronic phase of stroke [38].

\subsection{Neurogenesis, synaptogenesis and axonal remodeling}

In the adult brain, endogenous NPCs are present in the subgranular zone (SGZ) of the dentate gyrus and in the rostral SVZ of the lateral ventricles. These are perpetual sites of neurogenesis, although NPCs proliferate and differentiate at a lower rate under normal conditions than after injury [39]. NPCs from the SVZ are destined for the olfactory bulb, whereas those of the SGZ migrate into the neighboring granule layer of the dentate gyrus [40]. Under pathophysiological conditions of ischemia, neurogenesis is stimulated [41]. 
Stroke leads to a marked increase of NPC proliferation in the SVZ [39,42], and these neuroblasts may migrate toward to the lesion area, and some of them differentiate into new neurons to replace lost or damaged neurons [39]. Transgenic ablation of doublecortinexpressing NPCs shows that neurogenesis directly contributes to functional recovery after stroke [43]. The survival rate of these newly generated NPCs is poor and the differentiation rate is low $[39,44,45]$. However, various approaches have been investigated to promote survival of endogenous NPCs after stroke. Doeppner et al. fused heat shock protein 70 (Hsp70) to a cell-penetrating peptide derived from the human immunodeficiency virus TAT protein, to enhance postischemic survival of endogenous NPCs and to improve neurological function [46].

Neurogenesis and angiogenesis are coupled processes [15,38,47,48]. Cerebral endothelial cells activated by ischemia enhance NPC proliferation and differentiation, while NPCs isolated from the ischemic SVZ promote capillary tube formation of endothelial cells, and these coupled processes are mediated by VEGF receptor 2 (VEGFR2) [49]. These newly generated cells may also act as catalysts to promote brain plasticity and recovery [49].

Neural plasticity includes the changes of the number of neurons, dendritic and axonal arborization, spine density, synapse number and size, and receptor density [41]. In addition to neurogenesis, the brain has a capacity to alter the structure and function of neurons and to respond to microenvironmental changes [41]. This capacity is the neurobiological basis which determines the complexity and activity of neuronal networks and also contributes to recovery of function after CNS injury. After stroke, the CNS rearranges its neural networks to rapidly reorganize structure and function, including synaptogenesis [50] and axonal sprouting in brain and even in the spinal cord [51,52]. After stroke, motor cortex activity significantly increases in both contralateral and ipsilateral motor cortex [53]. Axonal outgrowth and dendritic plasticity may be mediated by the phosphoinositide 3-kinase/Akt/ glycogen synthase kinase-3 $\beta$ signaling pathway [54], and the spatial orientation and distribution of fibers may be controlled by Leucine-rich repeat and IgG domain containing protein 1 (Lingo1) and Nogo receptor 1 [51]. Remodeling of the corticospinal tract (CST) axons in the spinal cord after stroke and treatment with MSCs, also promote motor function recovery [36,52]. By anterograde tracing with biotinylated dextran amine (BDA) into cortex, and retrograde tracing with pseudorabies virus (PRV) carrying enhanced green fluorescent protein (PRV-152-EGFP) and monomeric red fluorescent protein (PRV-614-mRFP) injection into left or right forelimb extensor muscles, respectively, Liu et al. found EGFPpositive pyramidal neurons were increased in the intact cortex and contralesional axons sprouted into the denervated spinal cord [36]. Using a transgenic mouse with yellow fluorescent protein CST labeling in conjunction with trans-synaptic PRV retrograde tracing, they found remodeling of the CST contributes to spontaneous functional recovery after stroke [52]. Bone marrow-derived MSCs markedly increased CST fibers sprouting into the denervated spinal cord, and the enhanced interhemispheric, intracortical and denervated spinal cord connections were highly correlated with behavioral outcome after stroke [36,53]. Furthermore, in vivo and in vitro data suggest that MSCs increase activation of tPA, and downregulate plasminogen activator inhibitor-1 (PAI-1) in astrocytes, and thereby promote neurite outgrowth and subsequent functional recovery after stroke [55]. 


\subsection{Astrogenesis and oligodendrogenesis}

Astrocytes are a target in stroke intervention [41,56], since they closely interact with other brain parenchymal cells and endothelial cells, to maintain normal CNS function such as control and recovery of cerebral blood flow [57], and promote brain remodeling after stroke. By secretion of VEGF, astrocytes stimulate the formation of new blood vessels in the ischemic brain, and thus are strong contributors to neurorestorative processes. By facilitating extracellular glutamate and potassium removal during synaptic activity, astrocytes interact with neurons to regulate synaptic plasticity [58].

Massive activation of astrocytes and prominent reactive gliosis in the ischemic penumbra have complex effects on the BBB, the inflammatory response, and metabolic, excitotoxic and oxidative crises [59]. Attenuation of reactive gliosis by genetic ablation of glial fibrillary acidic protein (GFAP) and vimentin expressed by active astrocytes leads to increased ischemic infarction [56]. After stroke, astrocytes directly control the number and function of neuronal synapses and axonal sprouting via thrombospondins [60,61]. GFAPnull mice display enhanced long-term potentiation of population spike amplitude and excitatory postsynaptic potential slope, directly demonstrating that astrocytes modulate synaptic efficacy in the CNS [62]. Mice deficient in thromobospondin 1 and 2 exhibit synaptic density and axonal sprouting deficit associated with impaired motor function recovery after stroke [61]. Li et al. found that allogeneic transplantation of bone marrowderived MSCs improved neurological recovery and enhanced reactive astrocyte-related axonal remodeling [28]. Processes of astrocytes also remodeled from hypertrophic star-like to tadpole-like shape and oriented parallel to the ischemic regions. Axonal projections emanating from individual neurons exhibited an overall orientation parallel to elongated radial processes of reactive astrocytes [28]. Chondroitin sulfate proteoglycans are secreted by both astrocytes and oligodendrocytes to create a growth-repulsive microenvironment for axons and dendrites. Bone marrow-derived MSCs were shown to increase levels of glial cell line-derived neurotrophic factor (GDNF) and tPA, and reduce levels of PAI-1 from astrocytes and oligodendrocytes, and thus decrease proteoglycan levels to promote axonal plasticity under ischemic conditions [63]. Ding et al. recently demonstrated that MSC treatment of stroke stimulates sonic hedgehog (Shh) expression in the parenchymal cells and thereby increases tPA expression which promotes axonal remodeling and neurite outgrowth [64].

How do MSCs communicate with and alter the parenchymal cells to promote plasticity and restore neurological function post stroke? Recently, a new direct vehicle for cell-cell communication and alteration between MSCs and brain parenchymal cells has been investigated.

Exosomes are membrane microvesicles sized $40-100 \mathrm{~nm}$ in diameter and are secreted by a wide range of cell types [65]; they contain microRNA (miRNA) and messenger RNA (mRNA), which can be transferred between cells and affect the protein production of recipient cells, and thus play an important role in cell-to-cell communication [65]. MiRNAs are $18-25$ nucleotide, nonprotein coding transcripts that post-transcriptionally control gene expression [66]. For example, microRNA 133b (miR-133b) is related to regeneration of 
axons and neurons. Xin et al. provided the first demonstration that MSCs can communicate and transfer micro-RNA133b (miR-133b) to astrocytes and neurons via exosomes. MiR-133b downregulates expression of connective tissue growth factor (CTGF), a major inhibitor of axonal growth at injury sites, and thereby thins the glial scar, possibly reducing inhibitory proteoglycans and thereby benefits neurite outgrowth; in addition, miR-133b also downregulates RhoA protein expression which can enhance regrowth of the CST after injury [65]. Thus, exosomes expressed from the MSCs alter miRNA expression in parenchymal cells, which modulate an array of protein expression within parenchymal cells, which act in concert to contribute to neurite outgrowth [65].

Mature oligodendrocytes generate the myelin sheaths that enwrap axons, and thereby ensure functional and efficient neuronal signaling conduction [67]. Oligodendrocytes are vulnerable to ischemic stroke and their damage leads to demyelination, which contributes to neurologic functional deficits [37,67]. Whereas neurogenesis has been studied and garnered attention as a potential therapeutic target, the plasticity responses of oligodendrocytes post stroke have been demonstrated only recently [37]. Oligodendrocyte generation and function are critical for enhancement of white matter recovery and restoration of brain function. Recruited oligodendrocytes can remyelinate axons, raising the possibility of therapeutic intervention. Mature oligodendrocytes in the adult CNS are postmitotic and are unable to proliferate in response to injury [67]. Myelin repair depends on the oligodendrocyte progenitors cells (OPCs), which are abundant and widely distributed in the adult CNS. They are generated from Type B cells in the SVZ, are pervasive in the white and gray matter and are present in the lesion areas as well [67-69]. OPCs are comprised of functionally distinct groups that differ in their ability to respond to neuronal activity and to undergo differentiation, and OPCs experience injury following ischemia [68]. Using cell-type and region-specific genetic labeling methods, studies found that the number of OPCs originating in the SVZ significantly increased after injury. These OPCs may migrate into injury areas, such as the striatum and corpus callosum to differentiate into the pre- and mature myelinating oligodendrocytes, and participate in myelin repair in the lesions of adult brain [37]. However, OPC differentiation efficiency is severely compromised after injury, indicating the need for an intervention to promote their differentiation [69]. A series signaling pathways are involved in oligodendrogenesis after stroke, such as the Shh [37] and bone morphogenetic proteins (BMPs) [70]. Zhang et al. has demonstrated the endogenous OPCs become the potential therapeutic targets for the white matter remodeling post stroke [45]. They found that OPC proliferation was increased in the ipsilateral SVZ, striatum and corpus callosum at 7 days after stroke, and then gradually decreased in the SVZ, but remarkably increased in the ischemic striatum during $14-60$ days after stroke. These OPCs gave rise to mature oligodendrocytes in the ischemic striatum and corpus callosum at 30 and 60 days after stroke. Transplantation of MSCs derived from bone marrow or adipose tissue have been shown to promote functional recovery and oligodendrogenesis in rat post stroke [22,37], including promotion of OPC proliferation and increase of the myelin area. MSC treatment stimulates oligodendrogenesis by activation of the Shh pathway post stroke [37]. 


\subsection{Angiogenesis, vascular remodeling and BBB repair}

A sufficient blood supply through extensive vascularization is fundamental to healthy brain function. The BBB is an important regulator of brain homeostasis. BBB breakdown after stroke is associated with the primary insult and the secondary cell death as well [1]. Therefore, recovery from stroke involves mechanisms of vascular remodeling and BBB repair.

Angiogenesis, the formation of new vessels, plays an important role in the remodeling of ischemic brain tissue through enhanced perfusion [12], and it involves a series of factors, such as VEGF and its receptor, angiopoietin 1 (Ang1), SDF-1, brain-derived neurotrophic factor (BDNF) and GDNF [1,71]. The penumbra appears to provide a neurovascular niche into which both new neurons and vasculature are encouraged to grow [71]. Mitotic, genetic, and viral labeling and chemokine/growth factor gain-of-function and loss-of-function studies show that stroke induced newly born neurons to migrate from the SVZ into this unique neurovascular niche in penumbra, and closely associate with the remodeling vasculature within this neurovascular niche via vascular production of SDF-1 and Ang1 [42,71].

Cross-talk between oligodendrocytes and cerebral endothelium contributes to vascular remodeling as well. In the oligovascular niche, oligodendrocytes accelerate the angiogenic response and promote angiogenesis after white matter injury by secreting matrix metalloproteinase-9 (MMP-9) [72]. Trophic interactions between vessels and oligodendrocytes and OPCs play critical roles in white matter homeostasis which disturbed under diseased conditions [73].

A range of stem cells, such as human umbilical tissue-derived stem cells, human multipotent adult progenitor cells and human bone marrow-derived MSCs promote secretion of VEGF, expression of VEGF receptor (i.e., VEGFR2) and angiopoietins, and boost angiogenesis in the ischemic brain after stroke $[15,38,47]$. In addition, these angiogenic vessel themselves may produce factors that stimulate CNS remodeling after ischemia [48]. The newly formed vessels, in addition to tissue perfusion, provide their benefit from the expression of factors, such as BDNF, VEGF, VEGFR2 and matrix MMPs to remodel damaged tissues in the brain after ischemia, to form new synapses and to attract endogenous neuroblasts originating in the SVZ [48].

Transplanted EPCs restore the integrity and function of BBB via their angiogenic and vasculogenic properties [14]. MSCs also enhance angiogenesis and vascular integrity by increasing expression of Ang1 and its receptor Tie2, VEGF and receptor VEGFR2 [74].

\subsection{Immunomodulation}

The CNS is subject to immunological surveillance more than a strictly immune-privileged tissue [75]. However, the immune system activated at the acute ischemic phase plays an important role in the pathogenesis of stroke [76]. Microglia and endothelial cells are activated by hypoxia and reactive oxygen species (ROS), and a series of adhesion molecules are induced on endothelial cells, leukocytes and platelets, such as intercellular adhesion molecule-1 (ICAM-1), vascular adhesion molecules (VCAMs), selectins and integrins, the permeability of the BBB is altered [77]. As the ischemic cascade progresses, cell death leads 
to production of a number of proinflammatory molecules, mediators and cytokines, such as nitric oxide (NO), interferon- $\gamma$ (IFN- $\gamma$ ), interleukin-6 (IL-6), IL-17, and tumor necrosis factor (TNF), NADPH oxidase-derived superoxide released by T lymphocytes, macrophages, microglia and neutrophils, and further worsen the inflammatory response, the state of oxidative stress, leading to neural cell apoptosis and neurotoxic effects [76,78].

Immune cells and inflammation play "dual" roles in tissue protection, repair and reorganization. Immunosuppression may ameliorate the tissue damage in the acute phase and promotes migration and survival of endogenous stem cells [79]; however, local inflammation can attract stem cells to the infarct and produce growth factors [80]. This highlights the difficulties with approaches based on full-blown suppression of inflammation. Furthermore, the infectious complications of therapies suppressing the immune system and inflammation also need to be taken into account.

Transplanted cells have immunomodulatory effects post stroke via secretion of cytokines, which directly inhibit T-cell activation, reduce leukocyte infiltration into the brain and local inflammation, and modulate the immune response, and thereby promote neural recovery [81]. The secreted cytokines include transforming growth factor beta (TGF $\beta$ ), hepatocyte growth factor (HGF), HLA-G, prostaglandin ( $\left.\mathrm{PGE}_{2}\right)$, IL-10, indoleamine 2,3-dioxygenase (IDO), and IFN $\gamma$ [82]. Stimulation of specific toll-like receptors (TLRs) expressed on MSCs affects their immune modulating responses. Activation of TLRs is triggered by ischemic pathologies, promote proliferation and migration of MSCs as well as secretion of cytokines from MSCs which evoke an immunomodulation effect [83]. Secretion of immune modulating factors from MSCs depends on specific TLR-agonist engagement [82]. MSCs can be polarized by downstream TLR signaling into two homogenously acting phenotypes, TLR4-primed hMSCs mostly elaborate proinflammatory mediators, and TLR3-primed hMSCs express mostly immunosuppressive factors. In vitro allogeneic cocultures of TLR3primed MSCs with peripheral blood mononuclear cells (PBMCs) lead to suppressed Tlymphocyte activation, while permissive T-lymphocyte activation in coculture with TLR4primed hMSCs. This polarization concept elucidates the effect of TLR stimulation and its downstream consequences on the immune modulating properties of stem cells [82]. This immunomodulatory function of MSCs has also been found in clinical trials, such as graft versus host disease, Crohn's disease and organ transplantation. A more complete understanding of the immunology of stroke is needed to develop the targeted approaches to selectively suppress the deleterious effects of inflammation.

Studies also highlighted the immunomodulatory properties of NPCs $[84,85]$, although the mechanism underlying immunomodulation remains poorly understood. Immunomodulation by NPCs may relate to their capacity to inhibit the proliferation of lymph node-derived T cells, increase the proportion of regulatory $\mathrm{T}$ cells and express immune molecules such as TGF- $\beta$, inducible nitric oxide synthase (iNOS) and prostaglandin E2 [75,84].

Splenic immune cells are released from spleen by blood-borne catecholamines through the activation of adrenergic receptors and involve in stroke-induced neurodegeneration [86]. Some types of cell therapies, such as human umbilical cord blood cells reduce peripheral immune cells emanating from the spleen [86] and convert peripheral organs into bioreactors 
that are anti-inflammatory and proregenerative after a stroke, to reduce infarct volume. Treatment of stroke with human umbilical cord blood cells under conditions of splenectomy abrogated the restorative therapeutic effect of the cell treatment, strongly suggesting that the spleen and the immune system play a vital role in mediating the therapeutic effect of this form of cell-based therapy for stroke [86].

\section{Clinical translation and risk}

Cell-based treatment of stroke has shown great promise in preclinical ischemic stroke studies, including improvement of function by promotion of neurogenesis, axonal plasticity, remyelination and angiogenesis, and modulation of inflammation. However, to translate these exciting therapies from the laboratory into clinical use faces many challenges [87]. The careful evaluation of therapeutic opportunities and potential risks is a prerequisite for clinical use of stem cell therapy. Included among the required investigations as a precondition for clinical translation of cell-based therapies are analyses of the type of stem cells, their proliferation capacity, the route of administration, irreversibility of treatment, long-term survival of engrafted cells, tumor formation and unwanted immune responses.

Clinical trials for cell-based therapy in stroke may proceed with a myriad of cell therapy products developed from brain, blood, bone marrow and adipose tissue in early clinical development. The short-term (up to 1 year) and the long-term (up to 5 years) clinical followup studies show that infusion of autologous MSCs was feasible and safe, and improved functional recovery and survival in ischemic stroke patients [88,89]. No significant side effects were observed. Large efficacy trials and longtime follow-up studies are needed to advance cell therapy to clinical treatment. In addition to identifying the most appropriate cell type, other issues including optimal therapeutic window, delivery route, cell dose and patient selection need to be resolved.

\subsection{Window, times and route of transplantation}

Therapeutic effects may differ depending on different treatment window, since the pathophysiological conditions of ischemic brain change dramatically according to disease course. The optimal time to transplantation after a stroke will depend on the therapeutic cell type and treatment strategy. If the treatment strategy targets neuroprotection, acute delivery of the cells will be critical; however, some conditions in the acute phase should be taken into account, such as release of excitotoxic neurotransmitters, free radicals and proinflammatory mediators which may decrease survival of transplanted cells and inhibit endogenous neurorestoration. Brain repair and plasticity after the acute phase take place over several weeks to months. If the intended action of cell-based therapy is restoration, then later delivery would be pertinent, since repair events mostly occur in the first 2 to 3 weeks after ischemia, and transplantation cells at this time may be most beneficial for this plasticity [90]. However, if cell-based transplantation is delayed to several weeks after stroke, then formation of scar tissue may adversely affect implanted cells [90]. The beneficial effects for cell-based treatment were reported when treatments were initiated days, weeks or months after stroke [32,91]. Moreover, by comparing therapeutic efficacy of human umbilical tissue-derived cells following experimental stroke, multiple injections were not superior to a 
single injection. A single injection is more convenient, faster, requires lower number of cells and is most likely associated with lower risks [92].

Delivery routes, for example intracerebral, intravenous and intra-arterial injection, affect the outcome of cell-based therapy [13]. Systemic delivery is the obvious goal for administration of cell, such as MSCs and NPCs. However, relevant lung adhesion molecules on the cell surface of MSCs may be a therapeutic obstacle, although, there have been reports that therapeutic benefit may actually derive from factors generated in the lung from the adherence of exogenously administered MSCs [93].

\subsection{Cell type of transplantation}

Ethical issues and potentially tumorigenic concerns arise from the work involving FSCs and ESCs [94,95], with the first report of a donor derived human brain tumor from fetal NSC therapy [96]. Therefore, the bulk of studies are toward the use of alternative cells. Reprogramming human adult somatic cells (i.e., fibroblasts) to iPSCs has boosted the stem cell field leading to increasing development and scientific knowledge [19]. Injected iPSCs migrated to injured areas and exhibited neurodifferentiation, immunomodulation, reduction of the lesion volumes and recovery of sensorimotor function [97]. However, the potential tumorigenic and technical limitations of iPSCs may ultimately stop or restrict their use as a clinical treatment for stroke patients. Although SCNT and cell--cell fusions succeed to generate iPSCs by a small set of factors $[18,19]$, the generation of iPSCs appears difficult, and the current efficiency makes it impractical for widespread human clinical [16]. Moreover, iPSCs have been associated with a higher rate of tumor formation [98]. Since both stem cells and cancer cells have some similar features, such as long life span, selfrenewal ability, relative apoptosis resistance, and similar growth regulators and control mechanisms [99], tumor formation is often seen as a key obstacle to the safe use of stem cell-based medicinal products. Generation of iPSCs employs the use of retro- and lentiviruses, reprogramming factors and modification of cell genome [18,19], and all these factors contribute to affect the tumorigenic potential of transplanted iPSCs.

NPCs are an important cell source for the treatment of stroke, given their reported stimulation of neurogenesis, angiogenesis and immunomodulation [75,84,85,91]. Exogenously administered NPCs survive and integrate well into the local environment after transplantation; however, the survival rate of grafted NPCs is poor, and this may hamper its use as a cell-based therapy. Studies have been conducted to improve the survival rate of transplanted NPCs [13]. A Phase I NPC human study, Pilot Investigation of Stem Cells in Stroke (PISCES), to determine whether the transplantation of NPCs is feasible and safe is underway in Scotland.

In contrast, MSCs appear to have several important advantages over other stem cell lineages; in particular, they are nontumorigenic and have nonimmunogenic characteristics [23]. MSCs have long-lasting positive effects on recovery of neurological function and induce structural and molecular plasticity [41]. Intravenous transplantation of autologous bone marrow-derived MSCs is safe and feasible [88,89]; moreover, these cells can be rapidly expanded in autologous serum, reducing cell preparation time and the risk of transmissible disorders. The clinical trials with MSCs in regenerative approach have not 
reported major health concerns, such as acute infusional toxicity, organ system complications, infection, death or malignancy [23]. Since immune recognition of the administered cells is particularly important when the cells are nonautologous, MSCs have the advantage of immuneprivileged and low immunogenic potential. MSCs have been shown to suppress lymphocyte proliferation to allogenic or xenogenic antigens leading to acceptation of allogenic or xenotransplants in animal models $[28,83]$.

However, the limitations and potential risks of transplantation of MSCs need to be taken into consideration with comorbidities. Chen found that bone marrow-derived MSC treatment when administered intravenously one day post stroke did not improve functional outcome in diabetic animal subjects with stroke, due to increased mortality, BBB leakage and brain hemorrhage [100]. In addition, there may be a potential "bystander tumor formation" of MSCs [101], although MSCs are described as nontumorigenic. MSCs may migrate toward primary tumors, and then modulate tumor growth and metastasis by providing supportive factors. In addition, MSCs may reduce immune rejection of the tumor cells thus allowing continued tumor growth [101]. Therefore, there is a compelling need for additional studies for the extended time of follow-up, and the safety of MSCs and other cells are required.

\section{Conclusion}

Cell-based therapy has demonstrated benefits as a neurorestorative treatment of stroke. This therapy promotes neurological recovery after stroke by stimulating endogenous neural plasticity and remodeling of brain tissue. In addition, a later treatment window provides the cell-based treatment with a far wider patient base compared with thrombolytic therapy and neuroprotective agents. Here, we provided an overview of aspects of cell-based therapy for stroke, and we briefly addressed issues related to transplanted cell types, therapeutic benefits, mechanisms of actions and risks.

\section{Expert opinion}

Neuroprotective strategies cannot prevent or reverse tissue damage in clinical ischemic stroke treatment, and the limitations of thrombolytic therapy restricts wide application in the clinic. The interest in exogenous cell-based therapy is increasing based on observations that it robustly promotes cerebral plasticity and neurological recovery after stroke. Many candidates for cell-based therapy have been investigated during the last decade. Because of the large number of cell types, from adult to embryonic, from stem cells to somatic cells are candidates for treatment, well-designed investigations addressing the benefits and risks of each candidate are required to identify the optimal cell-based therapy for stroke.

Unlike the restricted population of cell replacement strategy required for specific diseases, for example dopamine neuron loss in Parkinson's disease, there is the complexity of the pathophysiological changes and multiple cell types have been lost after stroke; therefore, cell-based therapy for stroke should focus on stimulating and fostering neural plasticity of the entire CNS, with a focus on the intact nonischemic tissue.

Transplanted stem cells enter damaged brain and work as "biofactory," which continue to stimulate and amplify the endogenous restorative effects of the ischemic brain, including 
neurogenesis, synaptogenesis and axonal remodeling, astrogenesis and oligodendrogenesis, angiogenesis and vascular remodeling, BBB repair, as well as immunomodulation effects. All these factors interact in a highly dynamic way, facilitating temporally and spatially orchestrated responses of brain networks. In view of the complexity of the systems involved, cell-based therapy for stroke treatments that stimulate and amplify these endogenous restorative mechanisms may be superior to the one time pharmaceutical treatment. Experimental studies with cell-based therapy show significant functional improvement and evident restorative benefits. Pre- and clinical experience with cell-based therapy appears promising, and raises hope for the ischemic stroke patient. Essentially, cell therapy can treat all patients without limitation of rapid intervention, and concern of blood flow perfusion, in sharp contrast with thrombolytic therapy and neuroprotective agent delivery.

However, the reports from the experimental studies and clinical trials regarding the unwanted risks provoked by the use of cell-based therapy are also expanding. Before it becomes part of clinical regenerative medicine, questions of the potential risks of cell-based therapies have to be answered, and the mechanisms underlying the restorative effects of cellbased therapy should be further clarified before moving forward to use of these cells in clinical practice. The optimal efficacy of cell-based therapy is dependent on many factors, including the type of transplanted cell, dose and purity of cells, window of treatment, times of administration, route of cell delivery, characteristics of stroke patients, that is associated illnesses, and the need for immunosuppression. All these factors must be considered and carefully assessed. Adverse concerns of cell treatment include potential tumorigenic, lung adhesion and side effects when associated with vascular risk factors and illnesses (i.e., diabetes). Clearly, long-term follow-up and in-depth studies are required to determine whether the cell-enhanced recovery is sustained, and the safety and efficacy of the stem cell therapy must be fully evaluated, in order to optimize and translate the remarkable celltransplantation therapy to aid recovery in clinical stroke patients.

\section{Acknowledgments}

M Chopp was supported by the National Institutes of Health (AG037506).

\section{Bibliography}

Papers of special note have been highlighted as either of interest $(\bullet)$ or of considerable interest $(\bullet)$ to readers.

1. Zhang ZG, Zhang L, Tsang W, et al. Correlation of VEGF and angiopoietin expression with disruption of blood-brain barrier and angiogenesis after focal cerebral ischemia. J Cereb Blood Flow Metab. 2002; 22:379-92. [PubMed: 11919509]

2. Zhang RL, Chopp M, Zhang ZG, et al. E-selectin in focal cerebral ischemia and reperfusion in the rat. J Cereb Blood Flow Metab. 1996; 16:1126-36. [PubMed: 8898684]

3. Zhang L, Zhang ZG, Chopp M. The neurovascular unit and combination treatment strategies for stroke. Trends Pharmacol Sci. 2012; 33:415-22. [PubMed: 22595494]

4. de Los Rios la Rosa F, Khoury J, Kissela BM, et al. Eligibility for intravenous recombinant tissuetype plasminogen activator within a population: the Effect of the European Cooperative Acute Stroke Study (ECASS) III Trial. Stroke. 2012; 43:1591-5. [PubMed: 22442174] 
5. Ahmed N, Wahlgren N, Grond M, et al. Implementation and outcome of thrombolysis with alteplase 3-4.5 h after an acute stroke: an updated analysis from SITS-ISTR. Lancet Neurol. 2010; 9:866-74. [PubMed: 20667790]

6. Auriel E, Bornstein NM. Neuroprotection in acute ischemic stroke-current status. J Cell Mol Med. 2010; 14:2200-2. [PubMed: 20716132]

7. Ehrenreich H, Weissenborn K, Prange H, et al. Recombinant human erythropoietin in the treatment of acute ischemic stroke. Stroke. 2009; 40:e647-56. [PubMed: 19834012]

8. Morris GF, Bullock R, Marshall SB, et al. The Selfotel Investigators. Failure of the competitive Nmethyl-D-aspartate antagonist Selfotel (CGS 19755) in the treatment of severe head injury: results of two phase III clinical trials. J Neurosurg. 1999; 91:737-43. [PubMed: 10541229]

9. Lyden P, Shuaib A, Ng K, et al. Clomethiazole acute stroke study in ischemic stroke (CLASS-I): final results. Stroke. 2002; 33:122-8. [PubMed: 11779900]

10. Olsson SB. Stroke prevention with the oral direct thrombin inhibitor ximelagatran compared with warfarin in patients with non-valvular atrial fibrillation (SPORTIF III): randomised controlled trial. Lancet. 2003; 362:1691-8. [PubMed: 14643116]

11. Sherman DG, Bes A, Easton JD, et al. Use of anti-ICAM-1 therapy in ischemic stroke: results of the Enlimomab Acute Stroke Trial. Neurology. 2001; 57:1428-34. [PubMed: 11673584]

12. Hermann DM, Chopp M. Promoting brain remodelling and plasticity for stroke recovery: therapeutic promise and potential pitfalls of clinical translation. Lancet Neurol. 2012; 11:369-80. [PubMed: 22441198]

13. Doeppner TR, Ewert TA, Tonges L, et al. Transduction of neural precursor cells with TAT-heat shock protein 70 chaperone: therapeutic potential against ischemic stroke after intrastriatal and systemic transplantation. Stem Cells. 2012; 30:1297-310. [PubMed: 22593021]

14. Kaneko Y, Tajiri N, Shinozuka K, et al. Cell therapy for stroke: emphasis on optimizing safety and efficacy profile of endothelial progenitor cells. Curr Pharm Des. 2012; 18:3731-4. [PubMed: 22574986]

15. Mora-Lee S, Sirerol-Piquer MS, Gutierrez-Perez M, et al. Therapeutic effects of hMAPC and hMSC transplantation after stroke in mice. PLoS One. 2012; 7:e43683. [PubMed: 22952736]

16. Yu J, Thomson JA. Pluripotent stem cell lines. Genes Dev. 2008; 22:1987-97. [PubMed: 18676805]

17. Thomson JA, Itskovitz-Eldor J, Shapiro SS, et al. Embryonic stem cell lines derived from human blastocysts. Science. 1998; 282:1145-7. [PubMed: 9804556]

18. Yu J, Vodyanik MA, Smuga-Otto K, et al. Induced pluripotent stem cell lines derived from human somatic cells. Science. 2007; 318:1917-20. [PubMed: 18029452]

19. Takahashi K, Tanabe K, Ohnuki M, et al. Induction of pluripotent stem cells from adult human fibroblasts by defined factors. Cell. 2007; 131:861-72. [PubMed: 18035408]

20. Marcus AJ, Woodbury D. Fetal stem cells from extra-embryonic tissues: do not discard. J Cell Mol Med. 2008; 12:730-42. [PubMed: 18194447]

21. Koch P, Kokaia Z, Lindvall O, et al. Emerging concepts in neural stem cell research: autologous repair and cell-based disease modelling. Lancet Neurol. 2009; 8:819-29. [PubMed: 19679274]

22. Gutierrez-Fernandez M, Rodriguez-Frutos B, Ramos-Cejudo J, et al. Effects of intravenous administration of allogenic bone marrow- and adipose tissue-derived mesenchymal stem cells on functional recovery and brain repair markers in experimental ischemic stroke. Stem Cell Res Ther. 2013; 4:11. [PubMed: 23356495]

23. Lalu MM, McIntyre L, Pugliese C, et al. Safety of cell therapy with mesenchymal stromal cells (SafeCell): a systematic review and meta-analysis of clinical trials. PLoS One. 2012; 7:e47559. [PubMed: 23133515]

24. Daadi MM, Maag AL, Steinberg GK. Adherent self-renewable human embryonic stem cell-derived neural stem cell line: functional engraftment in experimental stroke model. PLoS One. 2008; 3:e1644. [PubMed: 18286199]

25. Sanberg PR, Eve DJ, Metcalf C, et al. Advantages and challenges of alternative sources of adultderived stem cells for brain repair in stroke. Prog Brain Res. 2012; 201:99-117. [PubMed: 23186712] 
26. Oki K, Tatarishvili J, Wood J, et al. Human-induced pluripotent stem cells form functional neurons and improve recovery after grafting in stroke-damaged brain. Stem Cells. 2012; 30:1120-33. [PubMed: 22495829]

27. Weimann JM, Charlton CA, Brazelton TR, et al. Contribution of transplanted bone marrow cells to Purkinje neurons in human adult brains. Proc Natl Acad Sci USA. 2003; 100:2088-93. [PubMed: 12576546]

28. Li Y, McIntosh K, Chen J, et al. Allogeneic bone marrow stromal cells promote glial-axonal remodeling without immunologic sensitization after stroke in rats. Exp Neurol. 2006; 198:313-25. [PubMed: 16455080]

29•. Li Y, Chen J, Wang L, et al. Treatment of stroke in rat with intracarotid administration of marrow stromal cells. Neurology. 2001; 56:1666-72. [PubMed: 11425931] The first evidence showing that MSCs from bone marrow improved neurological outcome after stroke.

30•. Chen J, Sanberg PR, Li Y, et al. Intravenous administration of human umbilical cord blood reduces behavioral deficits after stroke in rats. Stroke. 2001; 32:2682-8. [PubMed: 11692034] The first evidence showing that UCBCs improved neurological outcome after stroke.

31. Nystedt J, Anderson H, Tikkanen J, et al. Cell surface structures influence lung clearance rate of systemically infused mesenchymal stromal cells. Stem Cells. 2013; 31:317-26. [PubMed: 23132820]

32. Shen LH, Li Y, Chen J, et al. Therapeutic benefit of bone marrow stromal cells administered 1 month after stroke. J Cereb Blood Flow Metab. 2007; 27:6-13. [PubMed: 16596121]

33. Jaderstad J, Jaderstad LM, Li J, et al. Communication via gap junctions underlies early functional and beneficial interactions between grafted neural stem cells and the host. Proc Natl Acad Sci USA. 2010; 107:5184-9. [PubMed: 20147621]

34. Chen J, Li Y, Wang L, et al. Therapeutic benefit of intravenous administration of bone marrow stromal cells after cerebral ischemia in rats. Stroke. 2001; 32:1005-11. [PubMed: 11283404]

35. Shen LH, Li Y, Chen J, et al. One-year follow-up after bone marrow stromal cell treatment in middle-aged female rats with stroke. Stroke. 2007; 38:2150-6. [PubMed: 17525391]

36. Liu Z, Li Y, Zhang X, et al. Contralesional axonal remodeling of the corticospinal system in adult rats after stroke and bone marrow stromal cell treatment. Stroke. 2008; 39:2571-7. [PubMed: 18617661]

37. Zhang J, Li Y, Zhang ZG, et al. Bone marrow stromal cells increase oligodendrogenesis after stroke. J Cereb Blood Flow Metab. 2009; 29:1166-74. [PubMed: 19384336]

38. Zhang L, Li Y, Zhang C, et al. Delayed administration of human umbilical tissue-derived cells improved neurological functional recovery in a rodent model of focal ischemia. Stroke. 2011; 42:1437-44. [PubMed: 21493915]

39. Arvidsson A, Collin T, Kirik D, et al. Neuronal replacement from endogenous precursors in the adult brain after stroke. Nat Med. 2002; 8:963-70. [PubMed: 12161747]

40. Curtis MA, Kam M, Nannmark U, et al. Human neuroblasts migrate to the olfactory bulb via a lateral ventricular extension. Science. 2007; 315:1243-9. [PubMed: 17303719]

41. Pekna M, Pekny M, Nilsson M. Modulation of neural plasticity as a basis for stroke rehabilitation. Stroke. 2012; 43:2819-28. [PubMed: 22923444]

42. Kojima T, Hirota Y, Ema M, et al. Subventricular zone-derived neural progenitor cells migrate along a blood vessel scaffold toward the post-stroke striatum. Stem Cells. 2010; 28:545-54. [PubMed: 20073084]

43. Jin K, Wang X, Xie L, et al. Transgenic ablation of doublecortin-expressing cells suppresses adult neurogenesis and worsens stroke outcome in mice. Proc Natl Acad Sci USA. 2010; 107:7993-8. [PubMed: 20385829]

44. Zhang R, Zhang Z, Zhang C, et al. Stroke transiently increases subventricular zone cell division from asymmetric to symmetric and increases neuronal differentiation in the adult rat. J Neurosci. 2004; 24:5810-15. [PubMed: 15215303]

45. Zhang RL, Chopp M, Roberts C, et al. Ascl1 lineage cells contribute to ischemia-induced neurogenesis and oligodendrogenesis. J Cereb Blood Flow Metab. 2011; 31:614-25. [PubMed: 20736965] 
46. Doeppner TR, Nagel F, Dietz GP, et al. TAT-Hsp70-mediated neuroprotection and increased survival of neuronal precursor cells after focal cerebral ischemia in mice. J Cereb Blood Flow Metab. 2009; 29:1187-96. [PubMed: 19384335]

47. Chen J, Zhang ZG, Li Y, et al. Intravenous administration of human bone marrow stromal cells induces angiogenesis in the ischemic boundary zone after stroke in rats. Circ Res. 2003; 92:692-9. [PubMed: 12609969]

48. Chopp M, Li Y, Zhang ZG. Mechanisms underlying improved recovery of neurological function after stroke in the rodent after treatment with neurorestorative cell-based therapies. Stroke. 2009; 40:S143-5. [PubMed: 19064763]

49. Teng H, Zhang ZG, Wang L, et al. Coupling of angiogenesis and neurogenesis in cultured endothelial cells and neural progenitor cells after stroke. J Cereb Blood Flow Metab. 2008; 28:764-71. [PubMed: 17971789]

50. Dell MW, Lin CC, Harrison V. Stroke rehabilitation: strategies to enhance motor recovery. Annu Rev Med. 2009; 60:55-68. [PubMed: 18928333]

51. Li S, Overman JJ, Katsman D, et al. An age-related sprouting transcriptome provides molecular control of axonal sprouting after stroke. Nat Neurosci. 2010; 13:1496-504. [PubMed: 21057507]

52. Liu Z, Zhang RL, Li Y, et al. Remodeling of the corticospinal innervation and spontaneous behavioral recovery after ischemic stroke in adult mice. Stroke. 2009; 40:2546-51. [PubMed: 19478220]

53. Liu Z, Li Y, Zhang ZG, et al. Bone marrow stromal cells enhance inter- and intracortical axonal connections after ischemic stroke in adult rats. J Cereb Blood Flow Metab. 2010; 30:1288-95. [PubMed: 20125183]

54. Ueno Y, Chopp M, Zhang L, et al. Axonal outgrowth and dendritic plasticity in the cortical periinfarct area after experimental stroke. Stroke. 2012; 43:2221-8. [PubMed: 22618383]

55. Xin H, Li Y, Shen LH, et al. Increasing tPA activity in astrocytes induced by multipotent mesenchymal stromal cells facilitate neurite outgrowth after stroke in the mouse. PLoS One. 2010; 5:e9027. [PubMed: 20140248]

56. Li L, Lundkvist A, Andersson D, et al. Protective role of reactive astrocytes in brain ischemia. J Cereb Blood Flow Metab. 2008; 28:468-81. [PubMed: 17726492]

57. Mulligan SJ, MacVicar BA. Calcium transients in astrocyte endfeet cause cerebrovascular constrictions. Nature. 2004; 431:195-9. [PubMed: 15356633]

58. Pannasch U, Vargova L, Reingruber J, et al. Astroglial networks scale synaptic activity and plasticity. Proc Natl Acad Sci USA. 2011; 108:8467-72. [PubMed: 21536893]

59. Pekny M, Nilsson M. Astrocyte activation and reactive gliosis. Glia. 2005; 50:427-34. [PubMed: 15846805]

60. Allen NJ, Bennett ML, Foo LC, et al. Astrocyte glypicans 4 and 6 promote formation of excitatory synapses via GluA1 AMPA receptors. Nature. 2012; 486:410-14. [PubMed: 22722203]

61. Liauw J, Hoang S, Choi M, et al. Thrombospondins 1 and 2 are necessary for synaptic plasticity and functional recovery after stroke. J Cereb Blood Flow Metab. 2008; 28:1722-32. [PubMed: 18594557]

62. McCall MA, Gregg RG, Behringer RR, et al. Targeted deletion in astrocyte intermediate filament (Gfap) alters neuronal physiology. Proc Natl Acad Sci USA. 1996; 93:6361-6. [PubMed: 8692820]

63. Shen LH, Li Y, Gao Q, et al. Down-regulation of neurocan expression in reactive astrocytes promotes axonal regeneration and facilitates the neurorestorative effects of bone marrow stromal cells in the ischemic rat brain. Glia. 2008; 56:1747-54. [PubMed: 18618668]

64. Ding X, Li Y, Liu Z, et al. The sonic hedgehog pathway mediates brain plasticity and subsequent functional recovery after bone marrow stromal cell treatment of stroke in mice. J Cereb Blood Flow Metab. 2013 Epub ahead of print.

65. Xin H, Li Y, Buller B, et al. Exosome-mediated transfer of miR-133b from multipotent mesenchymal stromal cells to neural cells contributes to neurite outgrowth. Stem Cells. 2012; 30:1556-64. [PubMed: 22605481]

66. Bartel DP. MicroRNAs: genomics, biogenesis, mechanism, and function. Cell. 2004; 116:281-97. [PubMed: 14744438] 
67. McTigue DM, Tripathi RB. The life, death, and replacement of oligodendrocytes in the adult CNS. J Neurochem. 2008; 107:1-19. [PubMed: 18643793]

68. De Biase LM, Nishiyama A, Bergles DE. Excitability and synaptic communication within the oligodendrocyte lineage. J Neurosci. 2010; 30:3600-11. [PubMed: 20219994]

69. Kako E, Kaneko N, Aoyama M, et al. Subventricular zone-derived oligodendrogenesis in injured neonatal white matter in mice enhanced by a nonerythropoietic erythropoietin derivative. Stem Cells. 2012; 30:2234-47. [PubMed: 22890889]

70. Samanta J, Alden T, Gobeske K, et al. Noggin protects against ischemic brain injury in rodents. Stroke. 2010; 41:357-62. [PubMed: 20019326]

71. Ohab JJ, Fleming S, Blesch A, et al. A neurovascular niche for neurogenesis after stroke. J Neurosci. 2006; 26:13007-16. [PubMed: 17167090]

72. Pham LD, Hayakawa K, Seo JH, et al. Crosstalk between oligodendrocytes and cerebral endothelium contributes to vascular remodeling after white matter injury. Glia. 2012; 60:875-81. [PubMed: 22392631]

73. Arai K, Lo EH. Oligovascular signaling in white matter stroke. Biol Pharm Bull. 2009; 32:163944. [PubMed: 19801821]

74. Zacharek A, Chen J, Cui X, et al. Angiopoietin1/Tie2 and VEGF/Flk1 induced by MSC treatment amplifies angiogenesis and vascular stabilization after stroke. J Cereb Blood Flow Metab. 2007

75. Bonnamain V, Neveu I, Naveilhan P. Neural stem/progenitor cells as a promising candidate for regenerative therapy of the central nervous system. Front Cell Neurosci. 2012; 6:17. [PubMed: 22514520]

76. Iadecola C, Anrather J. The immunology of stroke: from mechanisms to translation. Nat Med. 2011; 17:796-808. [PubMed: 21738161]

77. Jin R, Yang G, Li G. Inflammatory mechanisms in ischemic stroke: role of inflammatory cells. J Leukoc Biol. 2010; 87:779-89. [PubMed: 20130219]

78. Kleinschnitz C, Kraft P, Dreykluft A, et al. Regulatory T cells are strong promoters of acute ischemic stroke in mice by inducing dysfunction of the cerebral microvasculature. Blood. 2013; 121:679-91. [PubMed: 23160472]

79. Erlandsson A, Lin CH, Yu F, et al. Immunosuppression promotes endogenous neural stem and progenitor cell migration and tissue regeneration after ischemic injury. Exp Neurol. 2011; 230:4857. [PubMed: 20685361]

80. Kelly S, Bliss TM, Shah AK, et al. Transplanted human fetal neural stem cells survive, migrate, and differentiate in ischemic rat cerebral cortex. Proc Natl Acad Sci USA. 2004; 101:11839-44. [PubMed: 15280535]

81. Broughton BR, Lim R, Arumugam TV, et al. Post-stroke inflammation and the potential efficacy of novel stem cell therapies: focus on amnion epithelial cells. Front Cell Neurosci. 2012; 6:66. [PubMed: 23335880]

82. Waterman RS, Tomchuck SL, Henkle SL, et al. A new mesenchymal stem cell (MSC) paradigm: polarization into a pro-inflammatory MSC1 or an Immunosuppressive MSC2 phenotype. PLoS One. 2010; 5:e10088. [PubMed: 20436665]

83. Uccelli A, Moretta L, Pistoia V. Mesenchymal stem cells in health and disease. Nat Rev Immunol. 2008; 8:726-36. [PubMed: 19172693]

84. Kokaia Z, Martino G, Schwartz M, et al. Cross-talk between neural stem cells and immune cells: the key to better brain repair? Nat Neurosci. 2012; 15:1078-87. [PubMed: 22837038]

85. Giusto E, Donega M, Cossetti C, et al. Neuro-immune interactions of neural stem cell transplants: from animal disease models to human trials. Exp Neurol. 2013; (13):S0014-4886. 00092-7. [PubMed: 23507035]

86. Ajmo CT Jr, Collier LA, Leonardo CC, et al. Blockade of adrenoreceptors inhibits the splenic response to stroke. Exp Neurol. 2009; 218:47-55. [PubMed: 19371742]

87. Wechsler L, Steindler D, Borlongan C, et al. Stem Cell Therapies as an Emerging Paradigm in Stroke (STEPS): bridging basic and clinical science for cellular and neurogenic factor therapy in treating stroke. Stroke. 2009; 40:510-15. [PubMed: 19095993]

88. Bang OY, Lee JS, Lee PH, et al. Autologous mesenchymal stem cell transplantation in stroke patients. Ann Neurol. 2005; 57:874-82. [PubMed: 15929052] 
89. Lee JS, Hong JM, Moon GJ, et al. A long-term follow-up study of intravenous autologous mesenchymal stem cell transplantation in patients with ischemic stroke. Stem Cells. 2010; 28:1099-106. [PubMed: 20506226]

90. Srivastava, RK.; Shankar, S. Stem cells and human diseases. Springer; New York, USA: 2012.

91. Andres RH, Horie N, Slikker W, et al. Human neural stem cells enhance structural plasticity and axonal transport in the ischaemic brain. Brain. 2011; 134:1777-89. [PubMed: 21616972]

92. Shehadah A, Chen J, Kramer B, et al. Efficacy of single and multiple injections of human umbilical tissue-derived cells following experimental stroke in rats. PLoS One. 2013; 8:e54083. [PubMed: 23342081]

93. Pati S, Gerber MH, Menge TD, et al. Bone marrow derived mesenchymal stem cells inhibit inflammation and preserve vascular endothelial integrity in the lungs after hemorrhagic shock. PLoS One. 2011; 6:e25171. [PubMed: 21980392]

94. Kalista T, Freeman HA, Behr B, et al. Donation of embryos for human development and stem cell research. Cell Stem Cell. 2011; 8:360-2. [PubMed: 21474099]

95. Park KD, Seong SK, Park YM, et al. Telomerase reverse transcriptase related with telomerase activity regulates tumorigenic potential of mouse embryonic stem cells. Stem Cells Dev. 2011; 20:149-57. [PubMed: 20486780]

96. Amariglio N, Hirshberg A, Scheithauer BW, et al. Donor-derived brain tumor following neural stem cell transplantation in an ataxia telangiectasia patient. PLoS Med. 2009; 6:e1000029. [PubMed: 19226183]

97. Chen SJ, Chang CM, Tsai SK, et al. Functional improvement of focal cerebral ischemia injury by subdural transplantation of induced pluripotent stem cells with fibrin glue. Stem Cells Dev. 2010; 19:1757-67. [PubMed: 20192839]

98. Kawai H, Yamashita T, Ohta Y, et al. Tridermal tumorigenesis of induced pluripotent stem cells transplanted in ischemic brain. J Cereb Blood Flow Metab. 2010; 30:1487-93. [PubMed: 20216552]

99. Werbowetski-Ogilvie TE, Bosse M, Stewart M, et al. Characterization of human embryonic stem cells with features of neoplastic progression. Nat Biotechnol. 2009; 27:91-7. [PubMed: 19122652]

100. Chen J, Ye X, Yan T, et al. Adverse effects of bone marrow stromal cell treatment of stroke in diabetic rats. Stroke. 2011; 42:3551-8. [PubMed: 21940967]

101. Lazennec G, Jorgensen C. Concise review: adult multipotent stromal cells and cancer: risk or benefit? Stem Cells. 2008; 26:1387-94. [PubMed: 18388305] 


\section{Article highlights}

- Cell-based therapy for stroke stimulates and amplifies endogenous restorative mechanisms in the CNS.

- Interactive restorative events including neurogenesis, angiogenesis, astrogenesis and oligodendrogenesis, and immunomodulation promoted by cell-based therapy contribute to the improvement in functional outcome after stroke.

- Optimal efficacy of cell-based therapy is dependent on many factors, including the administered cell type and dose, window of treatment, times of administration, route of cell delivery, characteristics of stroke patients and the need for immunosuppression.

- Well-designed investigations addressing the benefits and risks of each cell candidate are required to identify the optimal cell-based therapy for stroke.

- Adequate evaluation of the potential risks of promising cell-based therapy is a prerequisite for clinical application to stroke patients.

This box summarizes key points contained in the article. 


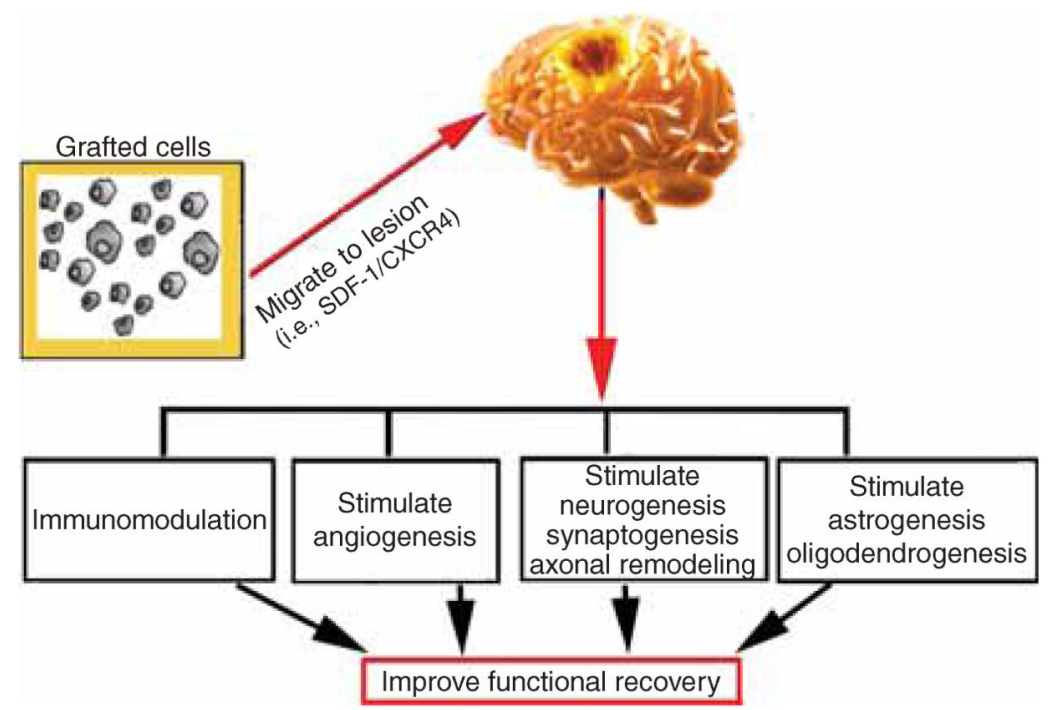

Figure 1. A schematic diagram of the mechanisms underlying the benefits of cell-based therapy for stroke After transplantation, grafted cells migrate into the lesion boundary zone by attracting factors, for example increased stromalderived factor-1 (SDF-1). Grafted cells promote neurogenesis, angiogenesis, astrogenesis and oligodendrogenesis, and immunomodulation. All these interactive restorative events contribute to the improvement in functional outcome. 